\title{
Spin polarized phases in strongly interacting matter: Interplay between axial-vector and tensor mean fields
}

\author{
Tomoyuki Maruyama* \\ College of Bioresource Sciences, Nihon University, Fujisawa 252-8510, Japan \\ Eiji Nakano ${ }^{\dagger}$ \\ Department of Physics, Kochi University, Kochi 780-8520, Japan \\ Kota Yanase ${ }^{\ddagger}$ and Naotaka Yoshinaga ${ }^{\S}$ \\ Department of Physics, Saitama University, Saitama City 338-8570, Japan
}

(Received 28 March 2018; revised manuscript received 17 May 2018; published 12 June 2018)

\begin{abstract}
The spontaneous spin polarization of strongly interacting matter due to axial-vector- and tensor-type interactions is studied at zero temperature and high baryon-number densities. We start with the mean-field Lagrangian for the axial-vector and tensor interaction channels and find in the chiral limit that the spin polarization due to the tensor mean field $(U)$ takes place first as the density increases for sufficiently strong coupling constants, and then the spin polarization due to the axial-vector mean field $(A)$ emerges in the region of the finite tensor mean field. This can be understood as making the axial-vector mean-field finite requires a broken chiral symmetry somehow, which is achieved by the finite tensor mean field in the present case. It is also found from the symmetry argument that there appear the type I(II) Nambu-Goldstone modes with a linear (quadratic) dispersion in the spin polarized phase with $U \neq 0$ and $A=0(U \neq 0$ and $A \neq 0)$, although these two phases exhibit the same symmetry breaking pattern.
\end{abstract}

DOI: 10.1103/PhysRevD.97.114014

\section{INTRODUCTION}

The discovery of the magnetars, the neutron stars with strong magnetic field of $\mathcal{O}\left(10^{15}\right) \mathrm{G}$, revives the important question about the origin of the strong magnetic field [1-4]. Recent studies have also revealed that magnetars possess not only the poloidal magnetic field but also the toroidal one, the strength of which is about $10^{2}$ times larger than the former $[5,6]$. There are several arguments about how such strong magnetic fields are generated and survive in the evolution of neutron stars [4], but a definite conclusion has yet to be made.

The spontaneous spin polarization or magnetization of the strongly interacting matter is one of the important issues in the relation to such a strong magnetic field. In an earlier work, Tatsumi [7] suggested the possibility of a ferromagnetic transition in quark matter interacting via a one-gluonexchange (OGE) force and showed that the maximum

\footnotetext{
${ }^{*}$ maruyama.tomoyuki@nihon-u.ac.jp

†e.nakano@kochi-u.ac.jp

*yanase@ nuclei.th.phy.saitama-u.ac.jp

yoshinaga@phy.saitama-u.ac.jp
}

Published by the American Physical Society under the terms of the Creative Commons Attribution 4.0 International license. Further distribution of this work must maintain attribution to the author(s) and the published article's title, journal citation, and DOI. Funded by SCOAP. magnetic field can reach $B \sim O\left(10^{15-17} \mathrm{G}\right)$ when the magnetar is a quark star.

In general, the ferromagnetic nature of dense matter manifests itself when a spin polarization of charged fermions, i.e., baryons or quarks, occurs collectively by their interactions, so that the spin degrees of freedom are a key ingredient. In the relativistic framework, we can consider two types of spinor bilinear forms as the spin density operator [8]: One is a spatial component of the axial-vector (AV) current operator, $\psi^{\dagger} \Sigma_{i} \psi\left(\equiv-\bar{\psi} \gamma_{5} \gamma_{i} \psi\right)$, and the other is that of the tensor (T) operator, $\psi^{\dagger} \gamma^{0} \Sigma_{i} \psi\left(\equiv-\frac{\epsilon_{i j k}}{2} \bar{\psi} \sigma_{j k} \psi\right)$, with $\psi$ being the Dirac field. These two become equivalent to each other in the nonrelativistic limit, while they are quite different in the ultrarelativistic limit (massless limit) [8]. When the expectation value of $\mathrm{AV}$ and/or T operators becomes finite, the spin polarization is realized. In fact, the T expectation value, $\left\langle\psi^{\dagger} \gamma^{0} \Sigma_{i} \psi\right\rangle$, directly reflects the magnetic effect through the electromagnetic coupling $\frac{Q}{2 m} \bar{\psi} \sigma_{\mu \nu} \psi F^{\mu \nu}$ (Gordon decomposition) for a particle with charge $Q$ and mass $m$, which is reminiscent of the Ising model under an external magnetic field. On the other hand, the finite AV expectation value, $\left\langle\psi^{\dagger} \Sigma_{i} \psi\right\rangle$, leads to a spin polarization, since the spatial components of the AV current correspond to the generators of rotations in the spinor representation of the Lorentz group.

In the previous studies, we have entirely relied on the mean-field approach, where we consider the AV and T 
channel interactions and make mean fields for them. As the origin of such interactions, the $\mathrm{AV}$-type interactions in quark matter can be derived from, e.g., the perturbative OGE interaction using the Fierz transformation, while the T-type interactions are expected to appear via the nonperturbative effects of QCD as seen in hadron-hadron effective interactions [9]. Then, the effective models of QCD should include both types of interactions. So far, the interplay between the spin polarization and other phases expected to appear in the high baryon-number density region have been studied, which includes the coexistence of the spin polarization and the color superconductivity [10-13] and the spatially homogeneous [14-18] and inhomogeneous chiral condensations $[19,20]$. From these studies, it is found that the AV- and T-type mean fields are affected differently by the dynamical chiral symmetry breaking: When the dynamical quark mass is zero, i.e., the chiral symmetry is restored, the AV-type spin polarized phase cannot appear, but the T-type one can. For instance, in the Nambu-Jona-Lasinio (NJL)type effective models, it has been demonstrated that the AVtype spin polarized phase can appear only in a narrow density region just inside the chiral condensed phase $[14,17,18]$, while the T-type spin polarized phase can exist in even higher density regions regardless of whether the dynamical quark mass is finite or not $[8,15-17]$. As will be shown below, this is because the T-type condensation itself breaks the chiral symmetry while the AV-type condensation respects them. So far, we have not known the spin polarized phase of systems including both the AV- and T-type interactions simultaneously, which is expected to exhibit new features of the spin polarization. Thus, in the present study, we investigate the interplay between them on the same footing and figure out the phase structure in terms of the coupling strengths of AV and $\mathrm{T}$ channels and of the baryon-number chemical potential at zero temperature in the chiral limit.

The paper is organized as follows. In the next section, we formulate a mean-field Lagrangian with $\mathrm{AV}$ and $\mathrm{T}$ mean fields and its thermodynamic potential at finite baryonnumber density and at zero temperature. In the Sec. III, after discussion of the relation between the spin polarization and the chiral symmetry, we search out the potential minimum to find the phase structure in the space of coupling constants. In Sec. IV, we demonstrate the change of phase structure with the chemical potential, employing a chiral model, and show that there appear two kinds of Nambu-Goldstone modes depending on the finiteness of the AV mean field. The last section is devoted to a summary and outlook.

\section{MEAN-FIELD APPROXIMATION AND THERMODYNAMIC POTENTIAL AT $T=0$ AND $\mu \neq 0$}

In this section, we briefly explain our formalism, which holds the flavor $S U(2)$ and the color $S U(3)$ symmetry. In addition, we consider only the spin-isospin saturated quark matter. We start with a general Lagrangian density including the spatial parts of $\mathrm{AV}$ and $\mathrm{T}$ fields,
$L=\bar{\psi}(i \not \partial-m) \psi+A_{i} \bar{\psi} \gamma_{5} \gamma_{i} \psi+U_{i j} \bar{\psi} \sigma_{i j} \psi-\frac{A_{i}^{2}}{g_{A}}-\frac{U_{i j}^{2}}{g_{U}}$,

where $\psi$ is the quark field, $m$ is the quark mass, $g_{A, U}$ is the effective coupling constants of the $\mathrm{AV}$ and $\mathrm{T}$ channels, $A_{i}=g_{A}\left\langle\bar{\psi} \gamma_{5} \gamma_{i} \psi\right\rangle$, and $U_{i j}=g_{U}\left\langle\bar{\psi} \sigma_{i j} \psi\right\rangle$. This Lagrangian is applicable for effective strong interactions included in, e.g., the NJL model, linear sigma model, and quark-quark interactions with screened gluons. In the isospin saturated system, the isospin dependent terms do not contribute to the mean field, and we omit them in the above Lagrangian.

Here, we assume only the third components of the mean fields, $A_{3}(=A)$ and $U_{12}(=U)$, to be nonzero and obtain the Dirac equation for the spinor $u(k, s)$ with momentum $\boldsymbol{k}=\left(k_{x}, k_{y}, k_{z}\right)$ and $\operatorname{spin} s$,

$$
\left[\boldsymbol{\alpha} \cdot \boldsymbol{k}+m+\Sigma_{z} A+\beta \Sigma_{z} U\right] u(\boldsymbol{k}, s)=\varepsilon_{k, s} u(\boldsymbol{k}, s) .
$$

The single-particle energy $\varepsilon_{k, s}$ is obtained as a solution of the characteristic equation for $\varepsilon$,

$$
\begin{aligned}
& \left(\varepsilon^{2}-E_{k}^{2}\right)^{2}-2 \varepsilon^{2}\left(A^{2}+U^{2}\right)-8 m A U \varepsilon-2 m^{2}\left(A^{2}+U^{2}\right) \\
& \quad+\left(A^{2}-U^{2}\right)^{2}-2\left(A^{2}-U^{2}\right)\left(k_{z}^{2}-k_{t}^{2}\right)=0,
\end{aligned}
$$

where $E_{k}=\sqrt{\boldsymbol{k}^{2}+m^{2}}$ and $k_{t}=\sqrt{k_{x}^{2}+k_{y}^{2}}$ is the magnitude of the transverse momentum normal to the polarization direction $z$. Since the above equations include the quark mass, it is easy to extend the present formulation to involve the chiral condensation in the same mean-field approximation. Nevertheless, we are interested in the high density region where the chiral condensation has already gone and intend to make discussion about the chiral symmetry transparent, so we take the chiral limit $m \rightarrow 0$ in the following calculations.

The thermodynamic potential is given by

$\Omega[A, U, \mu]=\Omega_{F}[A, U, \mu]+\Omega_{D}[A, U, \mu]+\frac{A^{2}}{g_{A}}+\frac{U^{2}}{g_{U}}$,

where $\Omega_{F}$ is the contribution from the matter component up to the Fermi surfaces and $\Omega_{D}$ is the contribution from the Dirac sea, given, respectively, by

$$
\begin{gathered}
\Omega_{F}[A, U, \mu]=N_{d} \sum_{s= \pm 1} \int_{k}\left(\varepsilon_{k, s}-\mu\right) \theta\left(\mu-\varepsilon_{k, s}\right), \\
\Omega_{D}[A, U, \mu]=-N_{d} \sum_{s= \pm 1} \int_{k} \varepsilon_{k, s},
\end{gathered}
$$

where the abbreviated notation $\int_{k} \equiv \int \mathrm{d} k^{3}$ is used, $N_{d}$ is the degeneracy factor, $\mu$ is the chemical potential. The singleparticle energies $\varepsilon_{k, s}$ in the chiral limit are given by

$$
\varepsilon_{p, s}=\sqrt{k_{z}^{2}+k_{t}^{2}+A^{2}+U^{2}+2 s \sqrt{k_{z}^{2} A^{2}+k_{t}^{2} U^{2}+A^{2} U^{2}} .}
$$




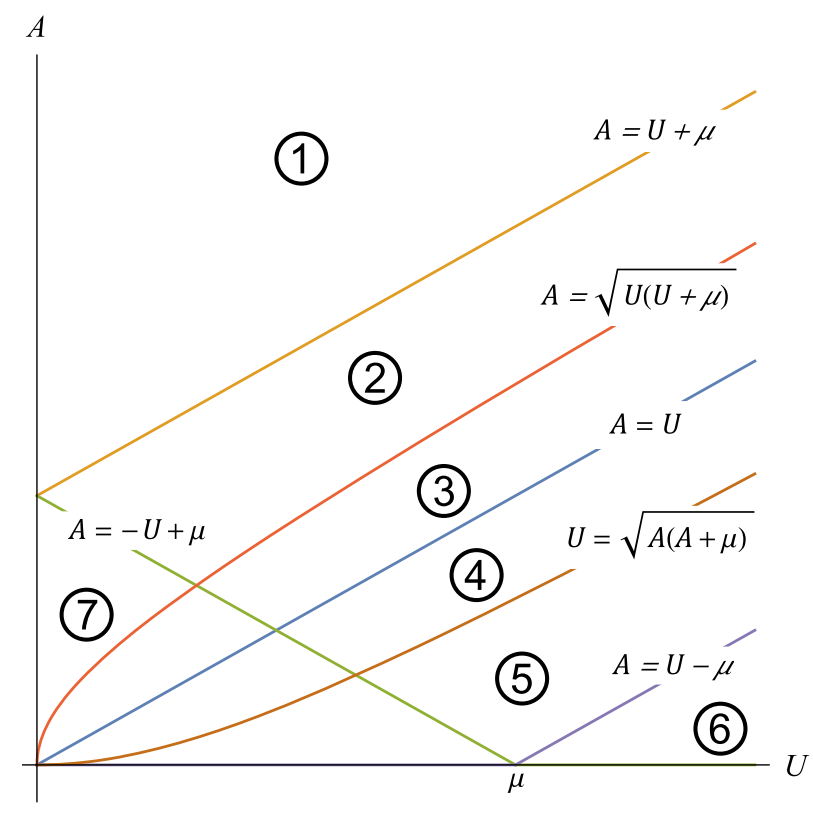

FIG. 1. (1)-(6) correspond to different topologies of Fermi surfaces for $s=-1$ as shown in Fig. 2, and the Fermi surface for $s=1$ becomes finite only in the region of (7): $A<-U+\mu$.
Note that the splitting of the energy spectrum by $s= \pm 1$ corresponds to the spin polarization due to the mean fields, and the magnitude of the mean fields is determined mainly by the matter contribution $\Omega_{F}$ as many-body effects, unlike the chiral condensation that comes from the Dirac sea contribution.

Although the above formulation can be applied to both hadronic and quark matters, we use $N_{d}=6$ (two flavors times three colors) and $\mu$ to be the quark chemical potential from now on. Also, from results of the preceding studies in which the spin polarization due to the AV or T mean fields is favored at higher densities where the chiral condensation already diminishes, we will neglect the Dirac sea contribution $\Omega_{D}$ of the thermodynamic potential in what follows.

\section{A. Fermi surfaces}

Since the single-particle energies, Eq. (7), are split and deformed by the mean fields, we take care of the topology of Fermi surfaces in the calculation of $\Omega_{F}$, especially for the $s=-1$ branch. The modification of the Fermi surface for $s=-1$ is classified for values of $A, U$, and $\mu$ in Fig. 1, and corresponding Fermi surfaces are shown in Fig. 2.
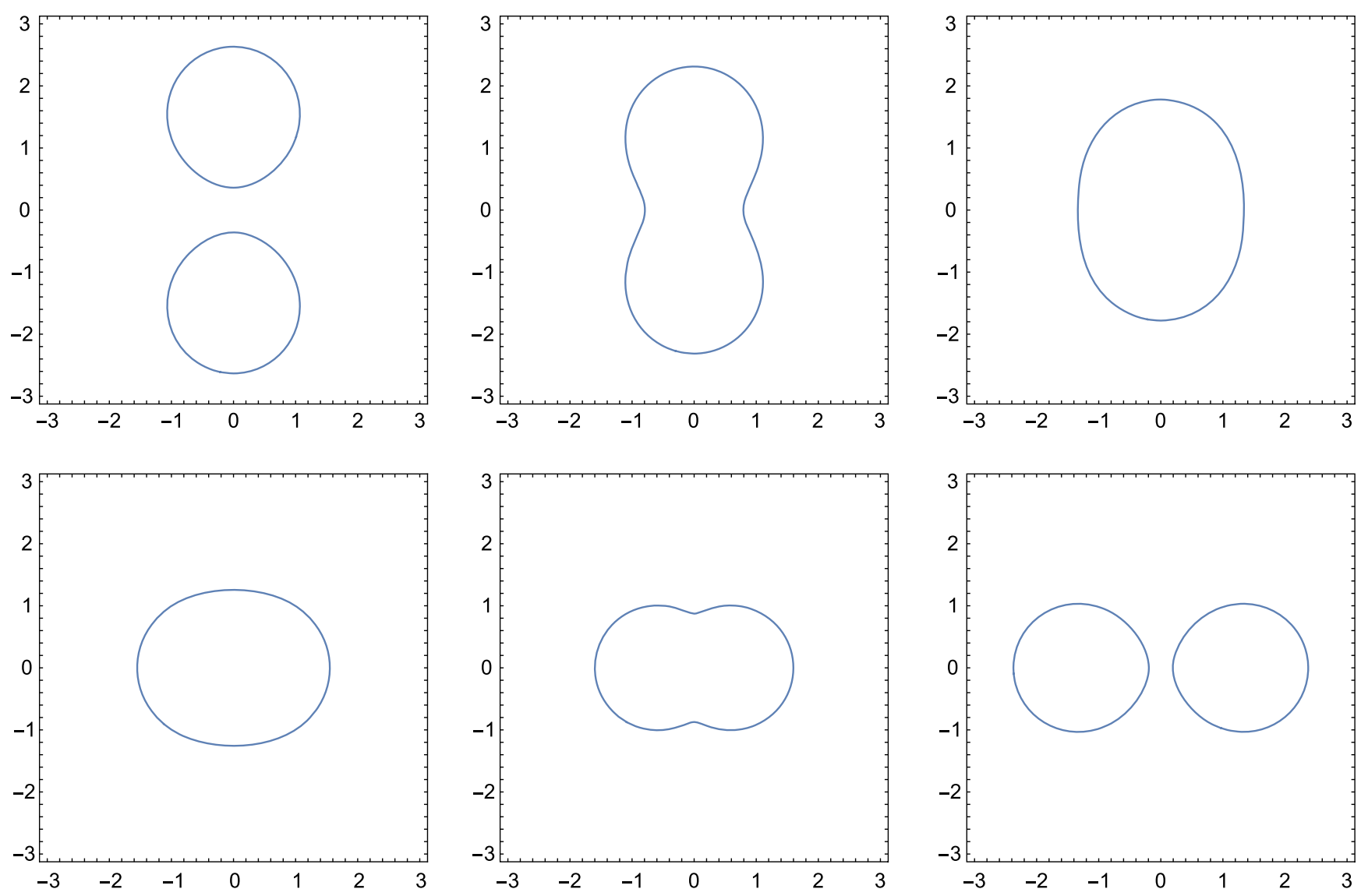

FIG. 2. Cross sections of Fermi surfaces in the $k_{y}-k_{z}$ plane for $s=-1$. The ordinate is $k_{z}$, and the abscissa is $k_{y}$. In the top row from left to right, (1) $\rightarrow$ (3), and in the bottom row from left to right, (4) $\rightarrow$ (6). Note that the Fermi surface is symmetric under the rotation around the $k_{z}$ axis, so the last one is a doughnut in full three dimensions. 


\section{B. Thermodynamic potential $\boldsymbol{\Omega}_{F}$ from Fermi seas}

The integral of $\Omega_{F}$ in the transverse direction $(y=$ $k_{x}^{2}+k_{y}^{2}$ ) can be done analytically:

$$
\begin{aligned}
\int_{k} \varepsilon_{k, s} \theta\left(\mu-\varepsilon_{k, s}\right) \\
=\frac{1}{4 \pi^{2}} \int \mathrm{d} k_{z} \int_{y_{1}}^{y_{2}} \frac{\mathrm{d} y}{2} \varepsilon_{k, s} \\
=\frac{1}{8 \pi^{2}} \int_{z_{1}}^{z_{2}} \mathrm{~d} k_{z}\left(\frac{\varepsilon_{k, s}^{2}}{6}+\frac{y+k_{z}^{2}+A^{2}-U^{2}}{2}\right) \varepsilon_{k, s} \\
\quad+s \frac{k_{z}^{2}\left(A^{2}-U^{2}\right)}{U} \\
\quad \times\left.\ln \left(\sqrt{y U^{2}+A^{2}\left(k_{z}^{2}+U^{2}\right)}+s U^{2}+U \varepsilon_{k, s}\right)\right|_{y_{1}} ^{y_{2}} .
\end{aligned}
$$

The integral ranges $z_{1,2}$ and $y_{1,2}$ are determined in accordance with the Fermi surfaces for $s= \pm 1$, and details of the calculations are given in Appendix A.

\section{PHASE STRUCTURE AND DISCUSSION}

The present study aims to figure out the phase structure in the space of coupling strengths and the chemical potential by searching out the minimum points of the thermodynamic potential $\Omega(A, U)$. To this end, it is heuristic to start with an argument on the properties of the mean fields: The spin polarization by $A \neq 0$ never occurs at $U=0$ in the chiral limit; that is, the $\Omega[A, U]$ is always stable against $A$ fluctuations at the origin in the $A-U$ space. This is because in a general AV-type mean field, appearing in the form of a mean-field interaction term $A_{i a} \bar{\psi} \gamma_{5} \gamma_{i} \tau_{a} \psi$ with $\tau_{a=0,1,2,3}$ being the identity or Pauli matrices in the isospin space, can be eliminated by a local chiral transformation $\psi \rightarrow e^{i \gamma_{5} A_{i a} \tau_{a} x_{i}} \psi$ through the derivative term [21], which costs zero energy in the chiral limit (by the redefinition of the spinor field). In other words, only in the case in which the chiral symmetry is broken, the net expectation value of the $\mathrm{AV}$ mean field can be generated. ${ }^{1}$ In the present case, a finite $U$ breaks the time reversal symmetry like in an external magnetic field and the $L-R$ symmetry as well; thus, it can invoke a finite $A$ for sufficiently strong couplings. From these observations, we set the strategy to get the phase structure as follows: We first find out the minimum point, $U_{\min }$, of the potential on the $U$ axis $(A=0)$ and then check if the second order derivative (curvature) in the $A$ direction is negative $\partial^{2} \Omega / \partial A^{2}<0$ or positive $\partial^{2} \Omega / \partial A^{2}>0$ at the minimum point. In the former case, the phase with $(A \neq 0, U \neq 0)$ can be realized, while in the latter case, a possible phase corresponds to $(A=0, U \neq 0)$, which, however, can be a

\footnotetext{
${ }^{1}$ Impacts of the axial anomaly to the axial-vector mean field at finite densities is neglected in this study.
}

local minimum. We have checked such situations and found no global minimum away from the $U$ axis in the present approximation.

As shown below, there exist some key points of the coupling constants, which determine the topology of the Fermi surfaces and signs of the potential curvatures on the $U$ axis. We give these relations at $N_{d}=6$ in the following:

(1) For $g_{U}>g_{U \text { crit }} \equiv \frac{4 \pi^{2}}{N_{d} \mu^{2}}=\frac{6.57974}{\mu^{2}}$, the potential curvature to the $U$ direction becomes negative, $\partial^{2} \Omega[A, U] / \partial U^{2}<0$, at $U=A=0$.

(2) For $g_{U} \geq g_{U 1} \equiv \frac{24 \pi}{N_{d} \mu^{2}}=\frac{12.5664}{\mu^{2}}, U_{\min } \geq \mu$, where $U_{\min }$ is the minimum point of the potential on the $U$ axis.

(3) For $g_{U} \geq g_{U 2} \equiv \frac{12.069141}{\mu^{2}}, U_{\text {min }} \geq U_{\text {cri }}=0.959993 \mu$, which is determined by $\partial_{U} \partial_{A}^{2} \Omega\left[0, U_{\text {cri }}\right]=0$.

(4) $g_{A 1}=\frac{12.4077}{\mu^{2}}$. Only if $g_{A} \geq g_{A 1} \equiv \frac{74.4462}{N_{d} \mu^{2}}=\frac{12.4077}{\mu^{2}}$, the potential curvature can be negative, $\partial^{2} \Omega / \partial A^{2}<0$ at $A=0$.

(5) When $g_{A 2}=\frac{12.5664}{\mu^{2}}, \partial^{2} \Omega / \partial A^{2}=0$ at $U=\mu$ and $A=0$. From these points, we obtained the boundary of the spin polarized phases as shown in Fig. 3. Its detailed derivation is given in Appendix $\mathrm{C}$. The phase structure is summarized as follows: The normal phase $(A=U=0)$ appears for small tensor couplings $g_{U} \leq g_{U \text { crit }}$. For $g_{U}>g_{U \text { crit }}$, the $\mathrm{T}$ mean field always becomes finite, but the finiteness of the $\mathrm{AV}$ mean field depends on the strength of the coupling $g_{A}$, which is bounded from below, i.e.,

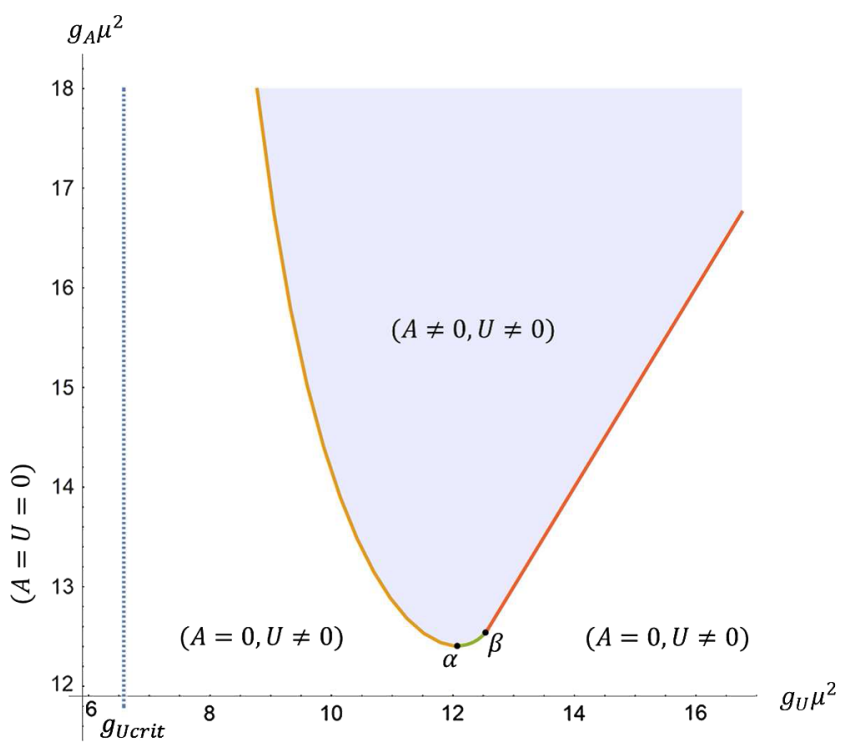

FIG. 3. Phase structure in the plane of axial-vector and tensor couplings, $g_{A}$ and $g_{U}$, normalized by the chemical potential $\mu$. The shaded region bounded from below by the solid curve corresponds to the $(A \neq 0, U \neq 0)$ phase. The other region is separated by the dotted line $\left(g_{U}=g_{U \text { crit }}\right): g_{U}>g_{U \text { crit }}$ corresponds to the $(A=0, U \neq 0)$ phase, and $g_{U} \leq g_{U \text { crit }}$ corresponds to the normal one $(A=U=0)$. Characteristic points are indicated by $\alpha=\left(g_{U 2}, g_{A 1}\right)$ and $\beta=\left(g_{U 1}, g_{A 2}\right)$. 
$g_{A}>g_{A 1}=12.4077 / \mu^{2}$. In strong coupling regions where $g_{A}, g_{U} \geq 12.5664 / \mu^{2}$, the two spin polarized phases are separated by the straight line $g_{A}=g_{U}$.

\section{APPLICATION TO AN EXTENDED NJL MODEL}

In the microscopic description of the strong interaction, perturbative vector-vector-type interactions, such as the single-gluon exchange interaction, do not generate T-type interactions even after the Fierz transformation, while effective models of the strong interaction are able to accommodate them as nonperturbative effects, e.g., the instanton induced interaction [22,23]. For an application, we employ an extended NJL model as an effective chiral model $[8,24]$,

$$
\begin{aligned}
L= & \bar{\psi} \not \partial \psi-G_{s}\left[(\bar{\psi} \psi)^{2}+\left(\bar{\psi} i \gamma_{5} \tau_{a} \psi\right)^{2}\right] \\
& -G_{A}\left[\left(\bar{\psi} \gamma_{\mu} \psi\right)^{2}+\left(\bar{\psi} \gamma_{5} \gamma_{\mu} \tau_{a} \psi\right)^{2}\right] \\
& -G_{U}\left[\left(\bar{\psi} \sigma_{\mu \nu} \psi\right)^{2}+\left(\bar{\psi} i \gamma_{5} \sigma_{\mu \nu} \tau_{a} \psi\right)^{2}\right] .
\end{aligned}
$$

Using the Fierz transformation [25], we can single out the relevant interaction terms (exchange channels) which are to be mean fields, showing only the spatial components of AV and $\mathrm{T}$ terms explicitly,

$$
L=\bar{\psi} \not \partial \psi+\frac{g_{A}}{2}\left(\bar{\psi} \gamma_{5} \gamma_{i} \psi\right)^{2}+\frac{g_{U}}{2}\left(\bar{\psi} \sigma_{i j} \psi\right)^{2}+\cdots,
$$

where $\frac{g_{A}}{2}=-\frac{1}{2} G_{s}-G_{A}$ and $\frac{g_{U}}{2}=-\frac{1}{8} G_{s}-G_{U}$. Taking the mean-field approximation as $A=A_{3}=g_{A}\left\langle\bar{\psi} \gamma_{5} \gamma_{3} \psi\right\rangle$ and $U=U_{12}=g_{U}\left\langle\bar{\psi} \sigma_{12} \psi\right\rangle$ for the spin polarization to the $z$ direction, with the other mean fields being vanished consistently as $A_{1}=A_{2}=U_{13}=U_{23}=0$, then we come back to the mean-field Lagrangian (1), and the discussions above can be reused. Here, we note that in general one can take an arbitrary relative angle between these directed mean fields, i.e., taking $A_{1} \neq 0$ and $A_{2} \neq 0$ in addition to $A_{3}$ and $U_{12}$, which breaks all rotational symmetries, but

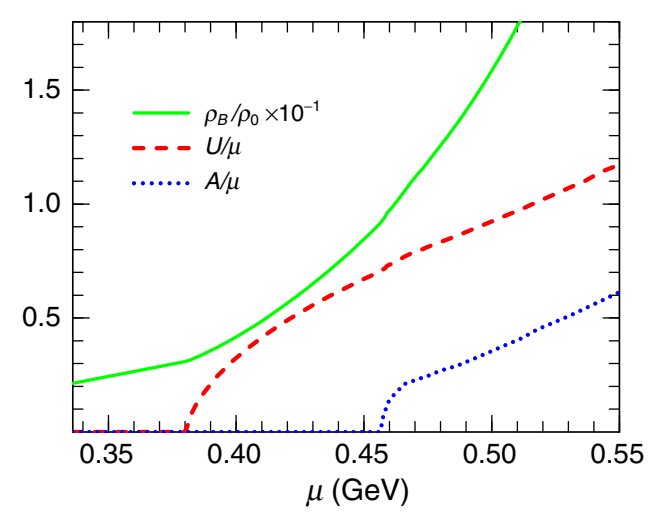

FIG. 4. The baryon-number density $\rho_{B} / \rho_{0}$, the axial-vector mean field $A / \mu$, and the tensor mean field $U / \mu$ as functions of quark chemical potential $\mu$ for $g_{A}=73.70\left(\mathrm{GeV}^{-2}\right)$ and $g_{U}=45.35\left(\mathrm{GeV}^{-2}\right) \cdot \rho_{0}=0.15 \mathrm{fm}^{-3}$ is the normal nuclear density. the stationary condition for the thermodynamic potential should prefer phases with the maximal residual symmetry, i.e., $A_{1}=A_{2}=0$.

Now, we demonstrate the change of phase structure with the chemical potential by numerical calculations. In Fig. 4, we plot the mean fields and the baryon-number density as functions of the quark chemical potential $\mu$, where numerical values of the coupling constants are fixed so as to reproduce the magnetic field expected at the core of magnetars [26,27], $g_{A}=73.70 \mathrm{GeV}^{-2}$ and $g_{U}=45.35 \mathrm{GeV}^{-2}$, which are of the same order of the coupling constants used in Refs. [14,17] and are an order of magnitude larger than in Refs. [15,16,18]. We will give details of the magnetic-field estimation around (22) in the last section. Since only the quark chemical potential brings the energy scale into the system, all quantities can be scaled by it. Therefore, as the $\mu$ increases, the corresponding trajectory in the phase diagram (Fig. 3) should be a straight line, i.e., $g_{A} / g_{U}=73.7 / 45.35=13 / 8$. The numerical result implies that the phase boundaries correspond to continuous phase transitions, and as discussed above, the AV mean field starts to get finite inevitably at a point where the $\mathrm{T}$ mean field is already finite.

\section{A. Low energy modes}

Once the phase structure is determined, the effective degrees of freedom are low energy excitations. Here, we discuss the Nambu-Goldstone modes upon the spin polarized phases. The symmetry $G$ of the system under the strong interaction and at a finite quark chemical potential is given by

$G=S O(3)_{\mathrm{rot}} \otimes S U(2)_{A} \otimes S U(2)_{V} \otimes U(1)_{B} \otimes S U(3)_{c}$,

where $S O(3)_{\text {rot }}$ represents the spatial rotation, $S U(2)_{A, V}$ is the axial and vectorial decomposition of the chiral flavor $S U(2)_{L, R}$ rotations, $U(1)_{B}$ is the baryon-number symmetry, and $S U(3)_{c}$ is the color gauge symmetry. The generators of the broken symmetries can be read off from the transformation of the order parameters: For $S U(2)_{A}$ chiral rotations, i.e., $\psi \rightarrow e^{i \frac{1}{2} \gamma_{5} \tau_{a} \pi^{a}} \psi$ where $\tau_{a=1,2,3}$ the Pauli matrices in the isospin space,

$$
\left[\gamma_{5} \tau_{a}, \gamma_{0} \sigma_{12}\right] \neq 0, \quad\left[\gamma_{5} \tau_{a}, \gamma_{0} \gamma_{5} \gamma_{3}\right]=0,
$$

and for the spatial rotations of the spinor, i.e., $\psi \rightarrow e^{i \Sigma_{k} \theta_{k}} \psi$ where $\Sigma_{k}=\frac{1}{2} \sigma_{i j} \epsilon^{i j k}$ with $\epsilon^{123}=1$ being the antisymmetric tensor,

$$
\begin{array}{r}
{\left[\Sigma_{3}, \gamma_{0} \sigma_{12}\right]=0, \quad\left[\Sigma_{1,2}, \gamma_{0} \sigma_{12}\right]=2 i \gamma_{0} \sigma_{13,23},} \\
{\left[\Sigma_{3}, \gamma_{0} \gamma_{5} \gamma_{3}\right]=0, \quad\left[\Sigma_{1,2}, \gamma_{0} \gamma_{5} \gamma_{3}\right]=\mp 2 i \gamma_{0} \gamma_{5} \gamma_{2,1} .}
\end{array}
$$

The commutations for the other generators of $G$ are vanishing. Thus, in the spontaneous spin polarized phases, the residual symmetry $H$ becomes 


$$
H=S O(2)_{\mathrm{rot}} \otimes S U(2)_{V} \otimes U(1)_{B} \otimes S U(3)_{c},
$$

where $S O(2)_{\text {rot }}$ reflects the invariance under a rotation around the $z$ axis. Note that the above symmetry breaking pattern is the same for the two spin polarized phases, i.e., for the $(A=0$, $U \neq 0)$ and $(A \neq 0, U \neq 0)$ phases.

Now, we examine the expectation values of commutators among generators of the broken symmetries, i.e., the generators of spatial rotations, and those of the $S U(2)_{A}$, defined, respectively, by

$$
\begin{gathered}
\Sigma_{k}(x) \equiv \psi^{\dagger}(x) \frac{1}{2} \sigma_{i j} \epsilon^{i j k} \psi(x)=-\bar{\psi}(x) \gamma_{5} \gamma_{k} \psi(x), \\
Q_{b}(x) \equiv \psi^{\dagger}(x) \frac{1}{2} i \gamma_{5} \tau_{b} \psi(x),
\end{gathered}
$$

where $k=1,2$ represent spatial directions of $x, y$ and $b=1,2,3$ represent the $S U(2)$ flavor triplet. We obtain the expectation values of commutators,

$$
\begin{aligned}
\left\langle\left[\Sigma_{1}(x), \Sigma_{2}(y)\right]\right\rangle & =i\left\langle\Sigma_{3}(x)\right\rangle V \delta^{(3)}(x-y) \\
& =-i A / g_{A} V \delta^{(3)}(x-y), \\
\left\langle\left[\Sigma_{i}(x), Q_{a}(y)\right]\right\rangle & =\left\langle\left[Q_{a}(x), Q_{b}(y)\right]\right\rangle=0,
\end{aligned}
$$

where $V$ is the volume of the system. From the above results, we can classify the number of NG bosons and their dispersion relations $[28,29]$ : In the $(A=0, U \neq 0)$ phase, there appear two Nambu-Goldstone (NG) bosons (type I) associated with broken spatial rotational symmetries, and their dispersion relations are linear in momentum for low energies as

$$
p_{0}=c|\boldsymbol{p}|,
$$

where $c$ is a coefficient (sound velocity), while in the $(A \neq 0, U \neq 0)$ phase, there is only a single NG boson (type II) associated with broken spatial rotational symmetries since Eq. (18) implies the broken generators $\Sigma_{1}$ and $\Sigma_{2}$ are canonically conjugate, i.e., they are not independent, and its dispersion relation can be quadratic as

$$
p_{0}=\tilde{c} \boldsymbol{p}^{2}
$$

with a different coefficient $\tilde{c}$. In addition, there must be three NG bosons (type I) associated with the broken $S U(2)_{A}$ flavor symmetries, which have a linear dispersion. The $U(1)_{A}$ symmetry is broken in reality by the quantum effect; thus, no associated NG boson exists. These NG bosons are relevant degrees of freedom for low energy dynamics, e.g., in scattering processes with photons and neutrinos [30-33].

\section{SUMMARY AND OUTLOOK}

We have studied the interplay between the axial-vector and tensor mean fields for a possible spontaneous spin polarization of the strongly interacting matter at finite baryon-number chemical potentials and at zero temperature in the chiral limit. It is found that as the chemical potential increases the $\mathrm{T}$ mean field $U$ becomes finite first at a critical point and breaks the chiral symmetries as well as the spatial rotation symmetries, while the AV mean field $A$ becomes finite only in the region of a finite $U$ because the finiteness of $A$ requires the chiral symmetry to be broken. All these phase boundaries correspond to continuous phase transitions. Furthermore, we classified the Nambu-Goldstone modes associated with broken rotational symmetries: There appear type I (II) NG modes with a linear (quadratic) dispersion in the phase of $U \neq 0$ and $A=0 \quad(U \neq 0$ and $A \neq 0$ ).

In relation to the magnetic field generated by these mean fields, we can estimate its strength as a magnetic moment density $M_{\mathrm{mag}}$ for the isospin saturated $u-d$ quark matter,

$$
M_{\mathrm{mag}}=\left(\frac{2}{3} \bar{n}_{u}-\frac{1}{3} \bar{n}_{d}\right) \frac{e \hbar}{2 m_{q}} \frac{\left\langle\bar{\psi} \sigma_{12} \psi\right\rangle}{\left\langle\bar{\psi} \gamma_{0} \psi\right\rangle} 3 \rho_{B},
$$

which amounts to $M_{\mathrm{mag}} \mu_{0} \simeq 1.1 \times 10^{18} \mathrm{G}$ for the quark mass $m_{q}=5 \mathrm{MeV} / \mathrm{C}^{2}, \mu_{0}$ for the vacuum permeability, and $\bar{n}_{u}=\bar{n}_{d}$ for the fraction of $u, d$-quark numbers $\bar{n}_{u}+\bar{n}_{d}=1$. The spin average $\frac{\left\langle\bar{\psi} \sigma_{12} \psi\right\rangle}{\left\langle\bar{\psi} \gamma_{0} \psi\right\rangle}=0.22$ is extracted from the extended NJL model (9) calculated at $\mu=$ $0.42 \mathrm{GeV}$ in Fig. 4, which gives the baryon-number density $\rho_{B}=5.54 \rho_{0}$ with $\rho_{0}=0.15 \mathrm{fm}^{-3}$ being the normal nuclear density, high enough to expect the chirally restored quark matter [25]. Although the in-medium permeability may be far from the vacuum one, the magnetic field estimated above is almost of the same order of magnitude expected at the core of magnetars, where the quark matter is assumed to develop [26,27]. Here, it should be noted that the inside of neutron stars is isospin asymmetric due to the charge neutrality and the beta equilibrium conditions, i.e., $\bar{n}_{d}>\bar{n}_{u}$, which may lead to a big reduction of the magnetic field in the present study. Even in such a situation, since the strong interaction is isospin symmetric, we can similarly consider isovector-type spin polarizations, e.g., $\left\langle\bar{\psi} \tau_{3} \sigma_{12} \psi\right\rangle$ as in Refs. $[15,16]$, or a linear combination of isoscalarand isovector-type spin polarizations as in Ref. [17]. In the isovector case, the relative sign of the spin polarization between $u$ and $d$ quarks flips due to the isospin Pauli matrix $\tau_{3}$; then, the charge average is replaced as $\left(\frac{2}{3} \bar{n}_{u}-\frac{1}{3} \bar{n}_{d}\right) \rightarrow\left(\frac{2}{3} \bar{n}_{u}+\frac{1}{3} \bar{n}_{d}\right)$; this replacement rather leads to an enhancement of the magnetic field.

In the present study, we have ignored the contribution from the Dirac sea in the thermodynamic potential. 
The four-fermion interactions employed here in an extended NJL model are nonrenormalizable theory, so one needs to introduce a cutoff and regularization scheme, and results may be changed quantitatively depending on them. However, the contribution of the Dirac sea can be absorbed (renormalized) into parameters (coupling constants of terms of the potential) in the mean-field approximation [34,35], and more importantly, in the isotropic regularization scheme in momentum space such as the proper-time regularization, the Dirac sea itself does not support the spin polarization, and only the finite density effects with deformed Fermi seas make the spin polarization possible as a many-body effect. Thus, the present result does not change at least qualitatively.

One of the other directions of further investigations is to figure out the finite mass effects on the spontaneous spin polarization. As discussed above, the chiral symmetry breaking is necessary to get a finite AV mean field. Once the chiral symmetry breaking terms, such as the current mass term, are introduced, they make $A$ easer to get finite even when $U=0$; thus, the present result may change so that the $(A \neq 0, U=0)$ phase appears for some parameter regions. In this respect, the axial anomaly, which breaks the $U(1)_{A}$ chiral symmetry explicitly, may affect the spin polarization as well $[36,37]$. We have also ignored the chiral condensation responsible for the dynamical chiral symmetry breaking, assuming very large baryon-number densities. To find more realistic phase structure, it is important to investigate the interplay among the spin polarization and the other orderings, e.g., the homogeneous/inhomogeneous chiral condensations in the moderate density region, and the color superconductivity in high density regions.

Although the spin polarization of the dense matter is not defined uniquely in the relativistic framework, we quantify it by AV and T mean fields in this study. The response to external stimulations may give other insight into the spin or magnetic properties of the strongly interacting matter, such as susceptibilities to external magnetic fields [18,38-41] and spatial rotations [42-45], both of which are related to neutron star physics.

\section{ACKNOWLEDGMENTS}

T. M. and E. N. are supported by Grants-in-Aid for Scientific Research from the Ministry of Education, Science and Culture of Japan through Grants No. 16K05360 and No. 17K05445 provided by JSPS.

\section{APPENDIX A: INTEGRATION UP TO FERMI SURFACES}

The explicit result of the integration (8) up to the Fermi surface for $s=-1$ is given by

$$
\begin{aligned}
& \int_{k} \varepsilon_{k,-1} \theta\left(\mu-\varepsilon_{k,-1}\right) \\
& =\frac{2}{8 \pi^{2}} \int_{z_{1}}^{z_{2}} \mathrm{~d} k_{z}\left(\frac{\varepsilon_{k,-1}^{2}}{6}+\frac{y+k_{z}^{2}+A^{2}-U^{2}}{2}\right) \varepsilon_{k,-1} \\
& -\frac{k_{z}^{2}\left(A^{2}-U^{2}\right)}{U} \\
& \quad \times\left.\ln \left(\sqrt{y U^{2}+A^{2}\left(k_{z}^{2}+U^{2}\right)}-U^{2}+U \varepsilon_{k,-1}\right)\right|_{y_{1}} ^{y_{2}}
\end{aligned}
$$

where

$y_{2}=-A^{2}-k_{z}^{2}+U^{2}+\mu^{2}+2 \sqrt{k_{z}^{2}\left(A^{2}-U^{2}\right)+U^{2} \mu^{2}}$,

$$
\begin{aligned}
y_{1}= & {\left[-A^{2}-k_{z}^{2}+U^{2}+\mu^{2}-2 \sqrt{k_{z}^{2}\left(A^{2}-U^{2}\right)+U^{2} \mu^{2}}\right] } \\
& \times \theta(U-\sqrt{A(A+\mu)}) \\
& \times \theta\left(k_{z}-\theta(A-U+\mu) \sqrt{(A-U+\mu)(A+U+\mu)}\right),
\end{aligned}
$$

$$
\begin{gathered}
z_{2}=\sqrt{(A-U+\mu)(A+U+\mu)} \theta(\sqrt{A(A+\mu)}-U) \\
+\frac{U \mu}{\sqrt{U^{2}-A^{2}}} \theta(U-\sqrt{A(A+\mu)}), \\
z_{1}=\sqrt{(A-U-\mu)(A+U-\mu)} \theta(A-U-\mu) .
\end{gathered}
$$

The integration up to the Fermi surface for $s=1$ is given by

$$
\begin{aligned}
\int_{k} \varepsilon_{k, 1} \theta\left(\mu-\varepsilon_{k, 1}\right) \\
=\frac{2}{8 \pi^{2}} \int_{0}^{z_{3}} \mathrm{~d} k_{z}\left(\frac{\varepsilon_{k, 1}^{2}}{6}+\frac{y+k_{z}^{2}+A^{2}-U^{2}}{2}\right) \varepsilon_{k, 1} \\
\quad+\frac{k_{z}^{2}\left(A^{2}-U^{2}\right)}{U} \\
\quad \times\left.\ln \left(\sqrt{y U^{2}+A^{2}\left(k_{z}^{2}+U^{2}\right)}+U^{2}+U \varepsilon_{k, 1}\right)\right|_{0} ^{y_{3}},
\end{aligned}
$$

where

$$
\begin{aligned}
y_{3}= & {\left[-A^{2}-k_{z}^{2}+U^{2}+\mu^{2}-2 \sqrt{k_{z}^{2}\left(A^{2}-U^{2}\right)+U^{2} \mu^{2}}\right] } \\
& \times \theta(\mu-U-A), \\
z_{3}= & \sqrt{(A-U-\mu)(A+U-\mu)} \theta(\mu-U-A) .
\end{aligned}
$$




\section{APPENDIX B: THE POTENTIAL CURVATURE WITH RESPECT TO $A$}

We calculate the second derivative of the thermodynamic potential with respect to $A$,

$$
\partial_{A}^{2} \int_{k} \varepsilon_{k, s} \theta\left(\mu-\varepsilon_{k, s}\right)=\int_{k} \partial_{A}^{2} \varepsilon_{k, s} \theta\left(\mu-\varepsilon_{k, s}\right)-\int_{k}\left(\partial_{A} \varepsilon_{k, s}\right)^{2} \delta\left(\mu-\varepsilon_{k, s}\right)-\partial_{A} \int_{k} \varepsilon_{k, s} \partial_{A} \varepsilon_{k, s} \delta\left(\mu-\varepsilon_{k, s}\right) ;
$$

then taking the limit of $A \rightarrow 0$ and noting that $\left.\partial_{A} \varepsilon_{k, s}\right|_{A \rightarrow 0}=0$, we obtain

$$
\left.\partial_{A}^{2} \int_{k} \varepsilon_{k, s} \theta\left(\mu-\varepsilon_{k, s}\right)\right|_{A \rightarrow 0}=\int_{k} \partial_{A}^{2} \varepsilon_{k, s} \theta\left(\mu-\varepsilon_{k, s}\right)-\left.\mu \partial_{A} \int_{k} \partial_{A} \varepsilon_{k, s} \delta\left(\mu-\varepsilon_{k, s}\right)\right|_{A \rightarrow 0},
$$

the second term of which cancels out with the second derivative of the density contribution,

$$
-\mu \partial_{A}^{2} \int_{k} \theta\left(\mu-\varepsilon_{k, s}\right)=\mu \partial_{A} \int_{k} \partial_{A} \varepsilon_{k, s} \delta\left(\mu-\varepsilon_{k, s}\right) .
$$

Then, the first term in (B2) can be calculated analytically, for $s=-1$, to be

$$
\begin{aligned}
\left.\int_{k} \partial_{A}^{2} \varepsilon_{-1} \theta\left(\mu-\varepsilon_{-1}\right)\right|_{A \rightarrow 0} & =\int \frac{\mathrm{d} z}{(2 \pi)^{2} U} \int \mathrm{d} \rho \frac{U(\rho-U)-z^{2}}{\sqrt{(U-\rho)^{2}+z^{2}}} \theta\left(\mu-\sqrt{(U-\rho)^{2}+z^{2}}\right) \\
& =\frac{\mu^{3}}{12 \pi^{2} U}\left[\frac{U}{\mu^{2}} \sqrt{\mu^{2}-U^{2}}+\cot ^{-1}\left(\frac{U}{\sqrt{\mu^{2}-U^{2}}}\right)+\frac{2 U^{3}}{\mu^{3}} \log \left(\frac{U}{\mu+\sqrt{\mu^{2}-U^{2}}}\right)\right] \theta(\mu-U)-\frac{\mu^{3}}{12 \pi U},
\end{aligned}
$$

while for $s=1$,

$$
\begin{aligned}
\left.\int_{k} \partial_{A}^{2} \varepsilon_{s} \theta\left(\mu-\varepsilon_{s}\right)\right|_{A \rightarrow 0} & =\frac{1}{(2 \pi)^{2} U} \int \mathrm{d} z \int \mathrm{d} \rho \frac{U(\rho+U)+z^{2}}{\sqrt{(U+\rho)^{2}+z^{2}}} \theta\left(\mu-\sqrt{(U+\rho)^{2}+z^{2}}\right) \\
& =\frac{\mu^{3}}{12 \pi^{2} U}\left[\frac{U}{\mu^{2}} \sqrt{\mu^{2}-U^{2}}+\cot ^{-1}\left(\frac{U}{\sqrt{\mu^{2}-U^{2}}}\right)+\frac{2 U^{3}}{\mu^{3}} \log \left(\frac{U}{\mu+\sqrt{\mu^{2}-U^{2}}}\right)\right] \theta(\mu-U) .
\end{aligned}
$$

Finally, the second derivative of $\Omega$ at $A=0$ reduces to

$$
\begin{aligned}
\left.\partial_{A}^{2} \Omega\right|_{A \rightarrow 0} & =\left.N_{d} \sum_{s} \int_{k} \partial_{A}^{2} \varepsilon_{s} \theta\left(\mu-\varepsilon_{s}\right)\right|_{A \rightarrow 0}+\frac{2}{g_{A}} \\
& =\frac{N_{d} \mu^{3}}{6 \pi^{2} U}\left[\frac{U}{\mu^{2}} \sqrt{\mu^{2}-U^{2}}+\cot ^{-1}\left(\frac{U}{\sqrt{\mu^{2}-U^{2}}}\right)+\frac{2 U^{3}}{\mu^{3}} \log \left(\frac{U}{\mu+\sqrt{\mu^{2}-U^{2}}}\right)\right] \theta(\mu-U)-\frac{N_{d}}{12 \pi} \frac{\mu^{3}}{U}+\frac{2}{g_{A}} .
\end{aligned}
$$

\section{APPENDIX C: THERMODYNAMIC POTENTIAL OF $U$ AT $A=0$ AND DETERMINATION OF PHASE BOUNDARY}

The thermodynamic potential at $A=0$ is analytically obtained as

$$
\begin{aligned}
\left(\frac{N_{d}}{24 \pi^{2}}\right)^{-1} \Omega[0, U]= & {\left[-\mu \sqrt{\mu^{2}-U^{2}}\left(2 \mu^{2}+3 U^{2}\right)-4 \mu^{3} U \tan ^{-1}\left(\frac{U}{\sqrt{\mu^{2}-U^{2}}}\right)\right.} \\
& \left.+U^{4} \log \left(\frac{\mu+\sqrt{\mu^{2}-U^{2}}}{U}\right)\right] \theta(\mu-U)-2 \pi \mu^{3} U \theta(U-\mu)+\frac{U^{2}}{g_{U}}\left(\frac{N_{d}}{24 \pi^{2}}\right)^{-1} .
\end{aligned}
$$




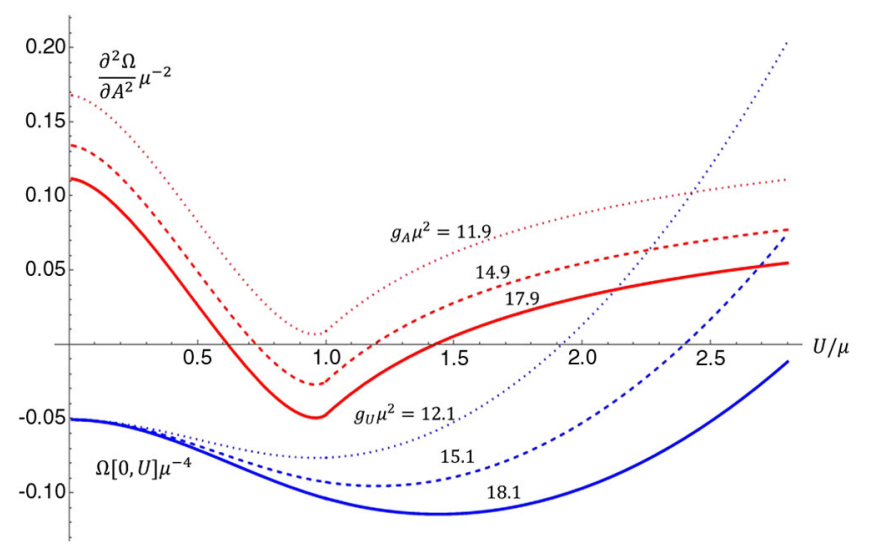

FIG. 5. The second derivative (B6) for $g_{A} \mu^{2}=g_{A 1} \mu^{2}+2.5=$ 14.9 (red dashed) and the potential (C1) for $g_{U} \mu^{2}=g_{U 1} \mu^{2}+2.5=$ 15.1 (blue dashed) are plotted as functions of $U$ in the unit of $\mu=1$. Red and blue solid (dotted) curves correspond to $g_{A} \mu^{2}=g_{A 1} \mu^{2}+$ $5.5=17.9$ and $g_{U} \mu^{2}=g_{U 1} \mu^{2}+5.5=18.1\left(g_{A} \mu^{2}=g_{A 1} \mu^{2}-0.5=\right.$ 11.9 and $\left.g_{U} \mu^{2}=g_{U 1} \mu^{2}-0.5=12.1\right)$, respectively.
The potential shape is shown in Fig. 5 together with the second derivative (B6) for some values of $g_{A}$ and $g_{U}$. As shown in the figure, the value of tensor mean field at the potential minimum, $U_{\min }$, increases monotonically with $g_{U}$, and zeros of the second derivative (B6) give two intersection points with the abscissa for larger values of $g_{A}$. If the $U_{\min }$ lies in between these intersection points, the phase with $U \neq 0$ and $A \neq 0$ is realized. Therefore, the phase boundary is determined by searching out $g_{U}$, for which $U_{\min }$ coincides with the intersection points for a given value of $g_{A}\left(\geq g_{A 1}\right)$. For smaller values of $g_{A}\left(<g_{A 1}\right)$, the intersection point disappears, meaning that the second derivative becomes positive everywhere. For larger values of $g_{U}$, corresponding to $U_{\min } \geq \mu$, the phase boundary is determined simply by $g_{A}=g_{U}$.
[1] For a review seeG. Chanmugam, Annu. Rev. Astron. Astrophys. 30, 143 (1992).

[2] P. M. Woods and C. Thompson, in Compact stellar X-ray sources, edited by W. Lewin and M. Van Der Klis, Soft gamma repeaters and anomalous X-ray pulsars:magfnetar candidates (Cambridge University Press, Cambridge, 2006); A. K. Harding and D. Lai, Rep. Prog. Phys. 69, 2631 (2006).

[3] C. Thompson and R. C. Duncan, Mon. Not. R. Astron. Soc. 275, 255 (1995).

[4] For reviews, R. Turolla, S. Zane, and A. L. Watts, Rep. Prog. Phys. 78, 116901 (2015); V. M. Kaspi and A. M. Beloborodov, Annu. Rev. Astron. Astrophys. 55, 261 (2017).

[5] J. Braithwaite and H. C. Spruit, Nature (London) 431, 819 (2004).

[6] K. Makishima, T. Enoto, J. S. Hiraga, T. Nakano, K. Nakazawa, S. Sakurai, M. Sasano, and H. Murakami, Phys. Rev. Lett. 112, 171102 (2014).

[7] T. Tatsumi, Phys. Lett. B 489, 280 (2000).

[8] T. Maruyama and T. Tatsumi, Nucl. Phys. A693, 710 (2001).

[9] For instance, P. Ring, Prog. Part. Nucl. Phys. 37, 193 (1996); E. V. Shuryak, The QCD Vacuum, Hadrons and Superdense Matter, 2nd ed., World Scientific Lecture Notes in Physics (World Scientific, Singapore, 2004), Vol. 71.

[10] E. Nakano, T. Maruyama, and T. Tatsumi, Phys. Rev. D 68 , 105001 (2003).

[11] Y. Tsue, J. da Providencia, C. Providencia, M. Yamamura, and H. Bohr, Prog. Theor. Exp. Phys. 2013, 103D01 (2013).

[12] Y. Tsue, J. da Providncia, C. Providncia, M. Yamamura, and H. Bohr, Prog. Theor. Exp. Phys. 2015, 13D02 (2015).

[13] H. Matsuoka, Y. Tsue, J. da Providencia, C. Providencia, and M. Yamamura, Phys. Rev. D 95, 054025 (2017).
[14] S. Maedan, Prog. Theor. Phys. 118, 729 (2007).

[15] Y. Tsue, J. de. Providencia, C. Providencia, and M. Yamamura, Prog. Theor. Phys. 128, 507 (2012).

[16] H. Matsuoka, Y. Tsue, J. da Providncia, C. Providncia, M. Yamamura, and H. Bohr, Prog. Theor. Exp. Phys. 2016, 053D02 (2016).

[17] T. Maruyama and T. Tatsumi, Phys. Rev. D 96, 096016 (2017).

[18] M. Morimoto, Y. Tsue, J. da Providencia, C. Providencia, and M. Yamamura, Int. J. Mod. Phys. E 27, 1850028 (2018).

[19] E. Nakano and T. Tatsumi, Phys. Rev. D 71, 114006 (2005).

[20] R. Yoshiike, K. Nishiyama, and T. Tatsumi, Phys. Lett. B 751, 123 (2015).

[21] Y. Nambu, J. Stat. Phys. 115, 7 (2004).

[22] T. Schaefer and E. V. Shuryak, Rev. Mod. Phys. 70, 323 (1998).

[23] G. W. Carter and D. Diakonov, Phys. Rev. D 60, 016004 (1999).

[24] For instance, T. Hatsuda and T. Kunihiro, Phys. Rep. 247, 221 (1994).

[25] For instance, S. P. Klevansky, Rev. Mod. Phys. 64, 649 (1992).

[26] D. Bandyopadhyay, S. Chakrabarty, and S. Pal, Phys. Rev. Lett. 79, 2176 (1997).

[27] R. H. Casali, L. B. Castro, and D. P. Menezes, Phys. Rev. C 89, 015805 (2014).

[28] H. Watanabe and H. Murayama, Phys. Rev. Lett. 108, 251602 (2012).

[29] Y. Hidaka, Phys. Rev. Lett. 110, 091601 (2013).

[30] J. N. Bahcall and R. A. Wolf, Phys. Rev. 140, B1452 (1965).

[31] B. L. Friman and O. V. Maxwell, Astrophys. J. 232, 541 (1979). 
[32] S. Reddy, M. Prakash, and J. M. Lattimer, Phys. Rev. D 58, 013009 (1998).

[33] T. Tatsumi and T. Muto, Phys. Rev. D 89, 103005 (2014).

[34] O. Scavenius, A. Mocsy, I. N. Mishustin, and D. H. Rischke, Phys. Rev. C 64, 045202 (2001).

[35] V. Skokov, B. Friman, E. Nakano, K. Redlich, and B.-J. Schaefer, Phys. Rev. D 82, 034029 (2010).

[36] J. Eser, M. Grahl, and D. H. Rischke, Phys. Rev. D 92, 096008 (2015).

[37] X. G. Huang, K. Nishimura, and N. Yamamoto, J. High Energy Phys. 02 (2018) 069.

[38] E. J. Ferrer and V. de la Incera, Phys. Rev. Lett. 102, 050402 (2009).
[39] E. J. Ferrer, V. de la Incera, I. Portillo, and M. Quiroz, Phys. Rev. D 89, 085034 (2014).

[40] K. Pal and A. K. Dutt-Mazumder, Phys. Rev. C 80, 054911 (2009).

[41] I. A. Shovkovy, Lect. Notes Phys. 871, 13 (2013).

[42] A. Vilenkin, Phys. Rev. D 20, 1807 (1979); 21, 2260 (1980).

[43] S. Ebihara, K. Fukushima, and K. Mameda, Phys. Lett. B 764, 94 (2017).

[44] M. N. Chernodub and S. Gongyo, J. High Energy Phys. 01 (2017) 136.

[45] Y. Liu and I. Zahed, arXiv:1710.02895. 